\title{
Development of a novel quantitative real-time assay using duplex mutation primers for rapid detection of Candida species
}

\author{
QIAN-FENG XIA $^{1 *}$, JIN-BO LIU $^{2 *}$, PING LIU $^{2}$, XI QIN $^{1}$, SHI-YUN QIAN $^{1}$ and ZHI-GUANG TU ${ }^{2}$ \\ ${ }^{1}$ Faculty of Tropical Medicine and Laboratory Medicine, Hainan Medical College, Haikou, \\ Hainan 570102; ${ }^{2}$ Key Laboratory of Laboratory Medical Diagnostics, Ministry of Education, \\ Faculty of Laboratory Medicine, Chongqing Medical University, Chongqing 400016, P.R. China
}

Received June 17, 2011; Accepted September 20, 2011

DOI: $10.3892 / \mathrm{mmr} .2011 .603$

\begin{abstract}
We developed a novel quantitative real-time PCR for quantitating Candida DNA based on the duplex mutation primer principle, in which a signal is generated by melting a duplex mutation primer during renaturation. The duplex mutation primers are much more specific than double-stranded DNA dyes such as SYBR-Green I and, unlike other probes, do not require the double-labeled synthesis of fluorophore and a quencher on the same molecule. A total of 176 clinical blood specimens were obtained from patients hospitalized in our hospital with clinically proven or suspected systemic Candida infection. The presence of DNA from pathogens in the Candida species was detected using real-time PCR targeting of an internal transcribed spacer region of a fungal gene. The assay exhibited a low limit of detection (10 CFU/ml of blood), an excellent reproducibility and specificity. Twenty-eight positive samples exhibited a wide range of Candida species loads, extending from 13 to $90,528 \mathrm{CFU} / \mathrm{ml}$ of blood. The sensitivity and specificity of the present assay were 100 and $97.4 \%$, respectively, compared with the results of blood culture. Our data suggest that this assay may be appropriate for use in clinical laboratories as a simple, low-cost and rapid screening test for the most frequently encountered Candida species.
\end{abstract}

\section{Introduction}

Advances in medical technologies and treatments, e.g., antimicrobial therapies, organ transplantation, and chemotherapy, have contributed to the dissemination of fungal infections $(1,2)$. Invasive candidiasis, a common cause of nosocomial infection, is a leading cause of infections among these patients and is also associated with a high rate of morbidity and mortality

Correspondence to: Dr Qian-Feng Xia, Faculty of Tropical Medicine and Laboratory Medicine, Hainan Medical College, 3 Yi Xue Yuan Road, Haikou, Hainan 570102, P.R. China

E-mail: xiaqianfeng@sina.com

${ }^{*}$ Contributed equally

Key words: Candida, fluorescent probes, diagnostics, real-time PCR, quantitative
(3). Candida albicans, C. tropicalis, C. glabrata, C. parapsilosis and $C$. krusei are the most common yeasts causing bloodstream infections, and these 6 species account for 95 to $98 \%$ of all blood yeasts $(4,5)$. To make matters worse, the prognosis for patients is relatively poor. The need for a rapid and accurate method for the detection of fungal pathogens has become imperative as the incidence of fungal infections has increased dramatically. However, most clinical laboratories rely on methods that employ phenotypic characteristics that can be time-consuming and not very accurate. Molecular diagnostic techniques such as nucleic acid detection by PCR are emerging as potentially more sensitive and rapid alternatives to conventional techniques for the diagnosis of invasive candidiasis (6). Our study focused on the development of a quantitative real-time PCR platform to detect Candida species DNA in clinical blood samples.

With rapid advances in molecular biology, combined with the public accessibility of microbial sequence data, we developed a rapid and simple assay with a high-throughput capability to detect all Candida species by using the duplex mutation primer technology. The real-time PCR assay based on the duplex mutation primer principle generates a signal by melting a duplex mutation primer during renaturation (7). The duplex mutation primers are much more specific than doublestrand DNA dyes such as SYBR-Green I and, unlike other probes (TaqMan, molecular beacons, scorpion primers, and amplifluor) $(8,9)$, do not require the double-labeled synthesis of fluorophore and a quencher on the same molecule (7).

To optimize our real-time PCR platform for the diagnosis of invasive candidiasis, we evaluated the sensitivity of this assay by adding $C$. albicans blastoconidia to blood, and also evaluated the specificities and the reproducibility and linear range of the novel assay. This optimized assay was tested with template DNA prepared directly from 176 blood samples from hematology or oncology patients with either disseminated candidiasis or suspected disseminated candidiasis. The performance of our assay was compared with the conventional microbiological techniques of blood culture and identification methods.

\section{Materials and methods}

Fungal strains and DNA extraction. Five reference yeast strains, Candida albicans ATCC 90028, C.tropicalis CDC 38, 

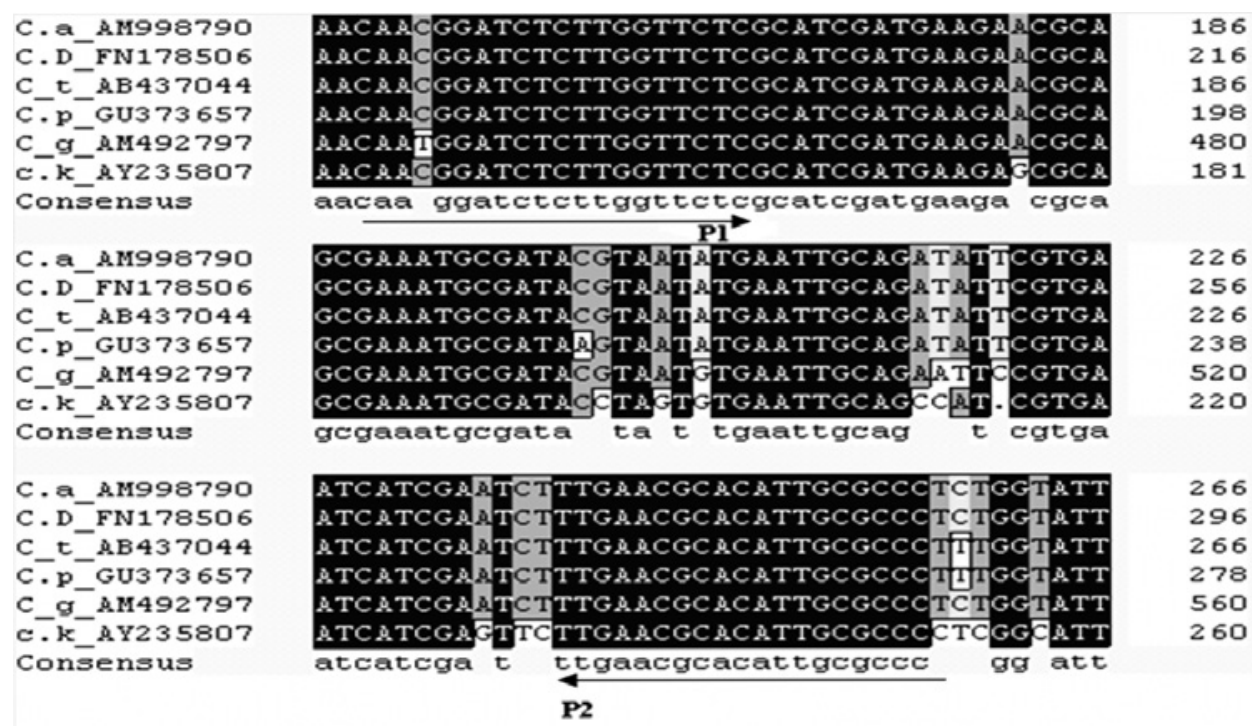

Figure 1. DNAMAN software multiple-sequence alignment of the ITS region amplified with primers P1 and P2 from C.albicans (AM998790), C.dubliniensis (FN178506), C. tropicalis (AB437044), C. parapsilosis (GU373657), C. glabrata (AM492797), and C. krusei (AY235807). (Designations in parentheses are GenBank accession numbers).

C. glabrata GAS 30, C.parapsilosis ATCC 22019, C. dubliniensis CBS 7988 and C. krusei ATCC 6258, were cultured on Sabouraud glucose-agar for $72 \mathrm{~h}$ at $30^{\circ} \mathrm{C}$. The identification of all clinical isolates and Candida species was confirmed by conventional morphological and physiological methods (10). Serial dilutions of $C$. albicans cells were prepared with sterile saline suspensions that were adjusted to a 0.5 McFarland standard (which is approximately $10^{6} \mathrm{CFU} / \mathrm{ml}$ ). DNA extraction with the QIAamp DNA blood kit (Qiagen, Hilden, Germany) was performed according to the manufacturer's instructions.

Clinical blood samples. During the prospective study period, a total of 176 clinical blood specimens obtained for routine microbiology diagnostic procedures from patients with clinically proven or suspected systemic Candida infection were retained for evaluation of the real-time PCR assays. Part of the material was cultured using standard cultivation methods. The remaining material $(2 \mathrm{ml})$ was stored at $-70^{\circ} \mathrm{C}$ until it was used for DNA extraction.

Primer design. GenBank was searched for sequences of the internal transcribed spacer (ITS) regions of Candida and phylogenetically related fungi. The published sequences were aligned using DNAMAN (Lynnon Corp., Quebec, Canada) software, and primers and probes were designed. Sequence alignments of the corresponding Candida species are shown in Fig. 1. BLAST search was performed to check the specificity of the DNA sequences of the primers.

The primers (P1: 5'-CAACAACGGATCTCTTGGTTCT CGC-3') and the fluorophore reverse primers (P2: 5'-FAMAGGGCGCAATGTGCGTTCAA-3') were used to amplify a 113 -bp fragment of ITS in the 6 Candida species. The fluorophore primers were quenched by partly complementary oligonucleotides (5'-TTGAACGCCCATTGCGCCCT -Dabcyl-3') of a single-base mismatched labeled with a quencher at the 3 '-end. All of the primers were synthesized, purified and labeled by Takara Ltd. Dalian, China.
Real-time PCR. Real-time PCR was performed with a Rotor-Gene 3000 (Corbett Robotics, Australia). The total volume of the real-time PCR was $20 \mu \mathrm{l}$ containing $5 \mu \mathrm{l}$ of target DNA, $4 \mu \mathrm{l}$ duplex mutation primers $(2.0 \mu \mathrm{M}$ of the fluorophore primer, $2.0 \mu \mathrm{M}$ of the quencher strand), $2.0 \mu \mathrm{M}$ of the reverse primer, $1.6 \mathrm{mM}$ of each $\mathrm{dNTP}, 1 \mathrm{U}$ of Taq polymerase and $2.0 \mathrm{mM} \mathrm{MgCl}_{2}$, adding distilled water to $20 \mu \mathrm{l}$. The cycling parameters were an initial denaturation at $93^{\circ} \mathrm{C}$ for $5 \mathrm{~min}, 40$ cycles at $93^{\circ} \mathrm{C}$ for $15 \mathrm{sec}$, and $49^{\circ} \mathrm{C}$ for $15 \mathrm{sec}$. Signal detection was performed at $49^{\circ} \mathrm{C}$ during each cycle. The 10 -fold serially diluted C. albicans blastoconidia used as external standards were used for the quantitation of DNA in the clinical samples.

Sensitivity and specificity. In order to evaluate the analytical sensitivity of the assays, whole blood from healthy volunteers was artificially spiked in a titration experiment with C. albicans blastoconidia to final concentrations of $10^{2}, 10^{1}$, and $5 \mathrm{CFU}$ per $\mathrm{ml}$ (in serial dilutions). DNA was extracted from the samples and analyzed. The analytical specificity of the assays was evaluated with DNA extracted from Candida and the other fungal clinical isolates of Aspergillus sydowii, Aspergillus versicolor, Aspergillus, Absidia corymbifera, Cunninghamella sp., Scedosporium apiospermum, Fusarium sp. and Scedosporium apiospermum.

Linearity range. To test the linearity range of the quantitative PCR, the stock of $C$. albicans genomic DNA at a concentration of $1 \times 10^{9} \mathrm{CFU} / \mathrm{ml}$, was diluted stepwise (1:10), and 4 to 6 replicates were tested per dilution.

Precision. To test the inter-run precision, amplification of DNA from C. albicans was performed $10^{5}, 10^{3}, 10^{2}$, and $10 \mathrm{CFU} / \mathrm{ml}$ of target in 5 parallel experiments. These 4 samples were repeatedly assayed 5 times in 5 independent experiments. The results of the quantitation, along with standard deviations and coefficients of variation (CVs) were 
A

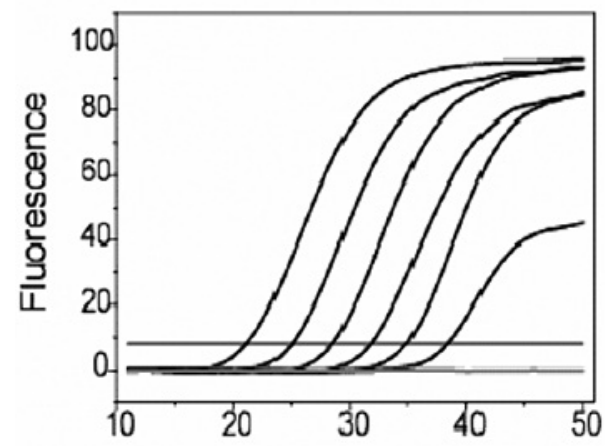

B

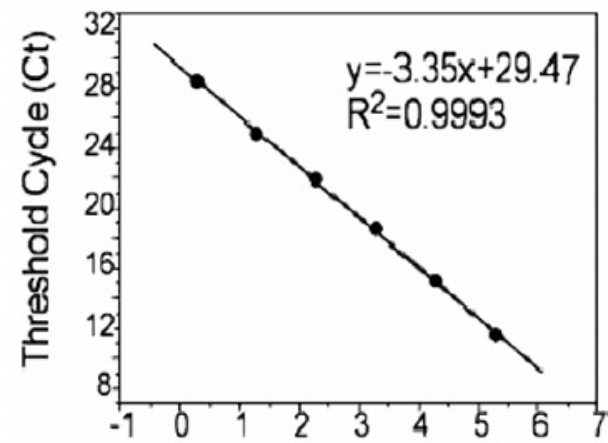

Figure 2. (A) Fluorescent curves of the standard dilution series. From left to the right, these six fluorescent curves from $10^{7}$ to $10^{2} \mathrm{CFU} / \mathrm{ml}$ are shown, and the negative control is represented by a horizontal black curve. (B) Standard curve used to calculate the concentration of Candida species DNA in unknown samples. The linear regression coefficient is close to 1.

evaluated. Intra-run precision was determined by measuring the amount of $C$. albicans DNA in the 4 samples mentioned above within one PCR run.

\section{Results}

Real-time PCR detection. The C. albicans blastoconidia dilution series of $10^{2} \sim 10^{7} \mathrm{CFU} / \mathrm{ml}$ was amplified in each PCR run (Fig. 2A). The cycle threshold $(\mathrm{Ct})$ was automatically set by the instrument software. The calibration curve of $\mathrm{Ct}$ vs. the template copy number showed linear regression analysis of the best-fit line and yielded a good correlation coefficient $\left(\mathrm{r}^{2}\right)$ of 0.999 (Fig. 2B). Analysis of reaction products by electrophoresis revealed that correct size of the reactions produced amplicons (Fig. 3).

Sensitivity and specificity. No signal was detected when the duplex mutation primers were used in these reactions with the negative controls. Fluorescence curves down to $10 \mathrm{CFU} /$ $\mathrm{ml}$ were detected. We decided to test the reproducibility of 5 and $1 \mathrm{CFU} / \mathrm{ml}$ to approach the limit of detection for the assay. Both 5 and $1 \mathrm{CFU} / \mathrm{ml}$ were not detected. The specificities of the assay was tested with a range of Candida strains and other fungal strains. The assay correctly identified $100 \%$ of the corresponding strains without cross-reaction with DNA purified from heterologous species.

Linearity. After various dilutions, the nucleic acid was extracted and the Candida albicans DNA was quantified. Linearity was observed from 10 up to $10^{9} \mathrm{CFU} / \mathrm{ml}$ in the sample.

Precision. The CVs of the CT values for these experiments with DNA from identical extractions were as follows: for $10^{5} \mathrm{CFU} / \mathrm{ml}, 3.51 \%$; for $10^{3} \mathrm{CFU} / \mathrm{ml}, 3.35 \%$; for $10^{2} \mathrm{CFU} / \mathrm{ml}$, $3.93 \%$ and for $10 \mathrm{CFU} / \mathrm{ml}, 4.42 \%$.

Quantitation of DNA in the clinical samples. The 176 clinical samples derived from patients irrespective of the presence of possible fungal infections were examined by standard cultivation and by real-time PCR. Twenty-eight samples tested displayed a signal by the novel assay analysis. The Candida

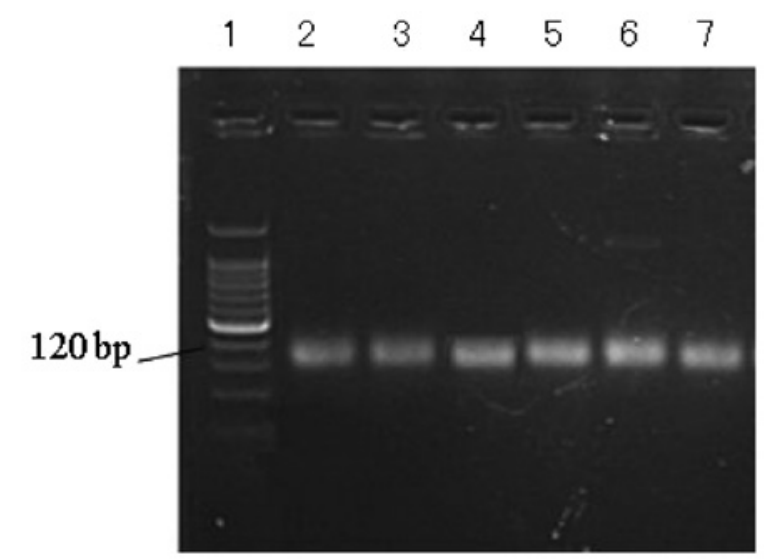

Figure 3. The PCR product was electrophoresed on an agarose gel and stained with ethidium bromide. Lane 1, DNA marker; lane 2, Candida albicans; lane 3, C.tropicalis; lane 2, C. glabrata GAS 30; lane 5, C. parapsilosis; lane 6,C.dubliniensis; lane 7,C. krusei. The products correspond to a $113 \mathrm{bp}$ DNA band.

species DNA load ranged between 13 and 90,528 CFU/ml of blood when the results were compared to those of a DNA standard. When the results of the culture were compared with the real-time PCR, 24 samples were found to be both culture positive and positive by real-time PCR. Four blood specimens from 4 patients which were culture negative were identified as containing Candida by the real-time assay (31, 42, 68 and $112 \mathrm{CFU} / \mathrm{ml}$ of blood). These 4 patients had previously received prophylactic fluconazole, which may have suppressed the Candida growth but did not completely eradicate the organism. Twenty-four of the 176 blood cultures were positive for Candida species: 15 for $C$. albicans, 5 for C. glabrata, 3 for $C$.tropicalis, 1 for C.krusei. Among the 28 blood specimens that were real-time PCR positive with the duplex mutation primers, 4 were culture negative for Candida species and 24 were culture positive for Candida species. Compared with the results of the blood culture, the sensitivity and specificity of the real-time PCR assay with the duplex mutation primers observed with blood samples were 100 and $97.4 \%$, respectively. 


\section{Discussion}

Candida bloodstream infections are associated with significant patient mortality and high health care costs. A relationship between a delay in the initial empiric antifungal treatment until blood culture results are known and the clinical outcome has been established (11). Rapid diagnosis of a Candida bloodstream infection may be the optimal method for avoiding delays in the treatment of this serious infection. Real-time PCR is a method that has been evaluated to more rapidly identify the presence of Candida species, as well as other microorganisms, from clinical specimens, including blood, spinal fluid and tissue biopsy specimens (12). The most extensively employed sequence-specific probes for real-time PCR are double-labeled, which make the design and preparation of probes difficult and expensive. Although hybridization probes (13), displacing probes (14) and Q-PNA primers (15) display certain advantages over double-labeled probes, hybridization probes and displacing probes, which need the 3'-phosphatation to block the amplification, are not real single-labeled probes, and the preparation of Q-PNA primers is complex. In contrast, duplex mutation primers are real single-labeled probes, which are easy to design, synthesize and apply in real-time PCR systems (7).

We described the development and validation of a novel quantitative real-time PCR assay that demonstrates superior performance characteristics in terms of the sensitivity, linearity, reproducibility and specificity. This assay has good clinical sensitivity at $10 \mathrm{CFU} / \mathrm{ml}$, with a minimum $10^{2}$ to $10^{9} \mathrm{CFU} / \mathrm{ml}$ linear dynamic range without the need for specimen dilution. The mandatory requirement of $100 \%$ specificity was achieved, as none of the controls, including the healthy donors and patients infected with the other clinical fungal strains, tested positive for Candida species. Five reference yeast strains, Candida albicans, C. tropicalis, C. glabrata, C. parapsilosis, C. dubliniensis and C. krusei, were tested positive. A similar performance was exhibited by another described quantitative TaqMan test for Candida (6).

In the present study, real-time PCR demonstrated a higher sensitivity than the culture. Of the 28 samples with proven or probable invasive candidiasis, the culture and PCR results were concurrently positive for 24 samples. There were 4 samples which were culture negative and PCR positive. The positive 'gold standard' for Candida detection is difficult to define, as most disseminated infections are not detected until death, and cultures may be falsely negative, for instance, due to the use of antifungal agents. In these cases, patients may have received antifungal therapy prior to the sampling, which may be a reason for these discrepancies. Discrepancies in the results of culture and PCR are well known and have already been previously observed (16). The detection of fungemia by conventional culture methods can be difficult which is generally attributed to very small fungal loads in clinical samples, and blood cultures have been shown to be positive in less than $50 \%$ of patients with chronic disseminated candidiasis $(17,18)$. Moreover, in contrast to a culture, qPCR results can be generated in 1 day. The greatest impact of the real-time PCR assay on routine diagnostic assays may be the short time period from the time of sample collection to the final diagnosis. In conclusion, a low-cost, rapid, sensitive and highly specific duplex mutation primer system was developed for detection of Candida.

\section{Acknowledgements}

This work was supported by grants Hj2010-20 and HjKj200524 from the Education Bureau of Hainan Province, China.

\section{References}

1. Kanj SS, Wolf KW, Madden J, Tapson V, Baz MA and Davis D: Fungal infections in lung and heart-lung transplant recipients, report of 9 cases and review of the literature. Medicine 75: 142-156, 1996.

2. Whimbey E, Kiehn T, Brannon P, Blevins A and Armstrong D: Bacteremia and fungemia in a patient with neoplastic diseases. Am J Med 82: 723-730, 1987.

3. Pittet D and Wenzel RP: Nosocomial bloodstream infections. Arch Intern Med 155: 1177-1184, 1995.

4. Pfaller MA, Messer SA, Hollis RJ, et al: Trends in species distribution and susceptibility to fluconazole among blood stream isolates of Candida species in the United States. Mycology 33: 217-222, 1999.

5. Chang HC, Chang JJ, Huang AH and Chang TC: Evaluation of a capacitance method for direct antifungal susceptibility testing of yeasts in positive blood cultures. J Clin Microbiol 38: 971-976, 2000.

6. Maaroufi Y, Heymans C, Bruyne DJM, et al: Rapid detection of Candida albicans in clinical blood samples by using a TaqMan-based PCR assay. J Clin Microbiol 41: 3293-3298, 2003.

7. Xia QF, Tu ZG, Liu JB, Qin X, Qian SY and Li P: Detection of PCR products using self-reporting duplex mutation primers. J Clin Pathol 62: 1046-1048, 2009.

8. Xia QF, Xu SX, Wang DS, et al: Development of a novel quantitative real-time assay using duplex scorpion primer for detection of Chlamydia trachomatis. Exp Mol Pathol 83: 119-124, 2007.

9. Tyagi $\mathrm{S}$ and Kramer FR: Molecular beacons: probes that fluoresce upon hybridization. Nat Biotechnol 14: 303-308, 1996.

10. Sullivan DK, Haynes J, Bille P, et al: Widespread geographic distribution of oral Candida dubliniensis strains in human immunodeficiency virus-infected individuals. J Clin Microbiol 35: 960-964, 1997.

11. Morrell M, Fraser VJ and Kollef MH: Delaying the empiric treatment of Candida bloodstream infection until positive blood culture results are obtained: a potential risk factor for hospital mortality. Antimicrob Agents chemother 49: 3640-3645, 2005.

12. Maaroufi Y, Bruyne DJM, Duchateau V, Georgala A and Crokaert F: Early detection and identification of commonly encountered Candida species from simulated blood cultures by using a real-time PCR-based assay. J Mol Diagn 6: 108-114, 2004.

13. Wittwer CT, Ririe KM, Andrew RV, et al: The LightCycler: a microvolume multisample fluorimeter with rapid temperature control. Biotechniques 22: 176-181, 1997.

14. Cheng J, Zhang Y and Li Q: Real-time PCR genotyping using displacing probes. Nucleic Acids Res 32: E61-E66, 2004.

15. Fiandaca MJ, Hyldig-Nielsen JJ, Gildea BD, et al: Self-reporting PNA/DNA primers for PCR analysis. Genome Res 11: 609-613, 2001.

16. Willinger B, Obradovic A, Selitsch B, et al: Detection and identification of fungi from fungus balls of the maxillary sinus by molecular techniques. J Clin Microbiol 41: 581-585, 2003.

17. Einsele H, Hebart H, Roller G, et al: Detection and identification of fungal pathogens in blood by using molecular probes. J Clin Microbiol 35: 1353-1360, 1997.

18. Borst A, Leverstein-Van Hall MA, Verhoef J and Fluit AC: Detection of Candida spp. in blood cultures using nucleic acid sequencebased amplification (NASBA). Diagn Microbiol Infect Dis 39: 155-60, 2001. 